\title{
Osteopathic Cranial Manipulative Medicine: Frontal and Parietal Lift Techniques
}

Dunia Mardini, OMS III; Nicole Peña, DO; Joel Talsma, MS; Stacey Pierce-Talsma, DO

\begin{abstract}
From the Touro University College of Osteopathic Medicine-CA in Vallejo.
\end{abstract}

Financial Disclosures: None

reported

Support: This video was produced by Touro University

College of Osteopathic

Medicine-CA.

Address correspondence to

Nicole Peña, DO, 1310 Club

Dr, Mare Island, Vallejo, CA

94592-1187.

Email: nicole.pena@tu.edu

Submitted

September 10, 2019;

accepted

October 31, 2019

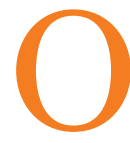
steopathic cranial manipulative medicine (OCMM) is a treatment modality that focuses on optimizing motion of the primary respiratory mechanism (PRM). This mechanism includes the anatomic and physiologic components of inherent motility of the central nervous system, fluctuation of the cerebrospinal fluid, articular mobility of the cranial bones, mobility of the cranial membranes, including the dura, and mobility of the sacrum between the ilia. ${ }^{1}$ Dysfunction of the PRM can occur antepartum, during childbirth, or be caused or compounded by traumatic injuries later in life. ${ }^{2}$

Many conditions may be helped by osteopathic manipulative treatment, including OCMM. These conditions include headache; orofacial pain; cranial nerve entrapment; vertigo; tinnitus; closed head trauma and concussion; temporomandibular joint dysfunction; ocular dysfunction, such as strabismus and astigmatism; various otolaryngologic complaints, such as eustachian tube dysfunction, otitis media, and chronic pharyngitis; dental pain and malocclusion; and plagiocephaly and feeding difficulties in children. ${ }^{1,3}$ Small pilot studies have also shown that OCMM and related craniosacral techniques may reduce symptoms associated with more severe neurologic and psychiatric disorders, such as cerebral palsy, posttraumatic stress disorder, and attention deficit/hyperactivity disorder. $^{4-6}$

In this OMT minute (video), we describe the frontal and parietal lift techniques. Associated structures include the frontal and parietal bones, which articulate with one another via the coronal suture. The paired frontal bones are separated by the metopic suture at birth, and the paired parietal bones are separated by the sagittal suture. The frontal bone articulates with the nasal, maxillary, zygomatic, lacrimal, ethmoid, and sphenoid bones, forming the roof of the orbital cavity. ${ }^{7}$ The parietal bones articulate with the occipital, temporal, sphenoid, and frontal bones, forming much of the superior and posterior aspects of the skull. ${ }^{7}$ Deep to these cranial bones lies the dura mater, a thick, double-layered fibrous membrane that adheres to the internal surface of the skull. Infoldings of the dural membrane separate the cranium into compartments and provide drainage pathways for venous blood and cerebrospinal fluid. ${ }^{7}$ The falx cerebri divides the right and left cerebral hemispheres, attaching to the internal surfaces of the frontal and ethmoid bones anteriorly and the internal surface of the occipital bone posteriorly. ${ }^{7}$ The tentorium cerebelli separates the cerebrum from the cerebellum and attaches to the internal surfaces of the sphenoid, temporal, occipital, and parietal bones. ${ }^{7}$

Osteopathic cranial manipulative medicine is a safe and effective treatment modality; however, there are instances when OCMM is contraindicated. Absolute contraindications include acute bleeds within the skull, skull fracture, and stroke. Relative contraindications include bleeding disorders, intracranial masses, and increased intracranial pressure. $^{1}$

The techniques we demonstrate is this video can be used to release articular, membranous, and ligamentous restrictions and strains in the frontal and parietal bones and associated structures. Additional treatment goals may include improvement in nerve function; improvement in venous, arterial, and lymphatic flow; and improvement in cerebrospinal fluid motion. ${ }^{1}$ Treatment may optimize motion of the PRM and address a variety of systemic diseases and symptoms. The treatments performed in this video are not intended to treat any specific clinical condition but are only one aspect of the diagnosis and treatment plan an osteopathic physician may use to address the whole patient. (doi:10.7556/jaoa.2019.139) 


\section{Acknowledgments}

We thank Jeff Reedy for contributions in video production and editing.

\section{References}

1. King HH. Osteopathic cranial manipulative medicine. In: Seffinger M, executive ed. Foundations of Osteopathic Medicine, Philosophy, Science, Clinical Applications and Research. 4th ed. Philadelphia, PA: Wolters Kluwer; 2018:885-906.

2. King HH, Lay EM. Osteopathy in the cranial field. In: Ward RC, executive ed. Foundations of Osteopathic Medicine. 2nd ed. Philadelphia, PA: Lippincott Williams \& Wilkins; 2003:985-1001.

3. The vault bones. Magoun HI. Osteopathy in the Cranial Field. 3rd ed. Indianapolis, IN: The Cranial Academy; 1976:170-179.
4. Duncan B, McDonough-Means S, Worden K, Schnyer R, Andrews J, Meaney FJ. Effectiveness of osteopathy in the cranial field and myofascial release versus acupuncture as complementary treatment for children with spastic cerebral palsy: a pilot study. J Am Osteopath Assoc. 2008;108(10):559-570.

5. Davis L., Hanson B., Gilliam S. Pilot study of the effects of mixed light touch manual therapies on active duty soldiers with chronic post-traumatic stress disorder and injury to the head. J Bodyw Mov Ther. 2016;20(1):42-51. doi:10.1016/j.jbmt.2015.03.006

6. Accorsi A, Lucci C, Di Mattia L, et al. Effect of osteopathic manipulative therapy in the attentive performance of children with attention-deficit/ hyperactivity disorder. J Am Osteopath Assoc. 2014;114(5):374-81. doi:10.7556/jaoa.2014.074

7. Moore KL, Dalley AF, Agur AM. Clinically Oriented Anatomy. 8th ed. Philadelphia, PA: Wolters Kluwer; 2014:829-886.

๑ 2019 American Osteopathic Association 